\title{
The effectiveness and cost evaluation of pain exposure physical therapy and conventional therapy in patients with complex regional pain syndrome type 1 . Rationale and design of a randomized controlled trial
}

\author{
Karlijn J Barnhoorn, Rob A B Oostendorp, Robert T M van Dongen, Frank P Klomp, Han Samwel, \\ Gert Jan van der Wilt, Eddy Adang, Hans Groenewoud, Henk van de Meent and Jan Paul M Frölke*
}

\begin{abstract}
Background: Pain Exposure Physical Therapy is a new treatment option for patients with Complex Regional Pain Syndrome type 1. It has been evaluated in retrospective as well as in prospective studies and proven to be safe and possibly effective. This indicates that Pain Exposure Physical Therapy is now ready for clinical evaluation. The results of an earlier performed pilot study with an $n=1$ design, in which 20 patients with Complex Regional Pain Syndrome type 1 were treated with Pain Exposure Physical Therapy, were used for the design and power calculation of the present study. After completion and evaluation of this phase III study, a multi-centre implementation study will be conducted. The aim of this study is to determine whether Pain Exposure Physical Therapy can improve functional outcomes in patients with Complex Regional Pain Syndrome type 1.

Methods/design: This study is designed as a single-blinded, randomized clinical trial. 62 patients will be randomized with a follow-up of 9 months to demonstrate the expected treatment effect. Complex Regional Pain Syndrome type 1 is diagnosed in accordance with the Brueh//International Association for the Study of Pain criteria. Conventional therapy in accordance with the Dutch guideline will be compared with Pain Exposure Physical Therapy. Primary outcome measure is the Impairment level SumScore, restricted version.
\end{abstract}

Discussion: This is the first randomized controlled study with single blinding that has ever been planned in patients with Complex Regional Pain Syndrome type 1 and does not focus on a single aspect of the pain syndrome but compares treatment strategies based on completely different pathophysiological and cognitive theories.

Trial registration: Clinical trials NCT00817128; National Trial Register NTR2090

\section{Background}

Complex Regional Pain Syndrome type 1 (CRPS-1) or reflex sympathetic dystrophy (RSD) is a chronic progressive condition of an extremity characterized by a variety of autonomic, sensory, motor and vasomotor symptoms, usually following injury.

It describes a variety of painful conditions following injury which appears regionally having a distal predominance of

\footnotetext{
* Correspondence: J.Frolke@chir.umcn.nl

Department of surgery 690, Radboud University Nijmegen Medical Centre, Nijmegen, Postbox 9101 6500, HB, The Netherlands
}

abnormal findings, exceeding in both magnitude and duration the expected clinical course of the inciting event [1].

It often results in impairments of neuromusculoskeletal and movement-related functions, sensory functions and pain, activity limitations and participation restrictions. It shows a variable progression in the course of time.

Clinical evaluation and diagnosis of CRPS- 1 are based on clinical assessment of signs and symptoms, in accordance with predetermined sets of diagnostic criteria [1].

Conventional therapy, consisting of pharmacological pain management and pain avoidance in functional management, has shown disappointing results in pain 
control and disabilities, and often leads to inactivity and social disintegration. Considerable impairments are still present over eight years after first CRPS-1 diagnosis [2].

Pain Exposure Physical Therapy (PEPT) is a new and promising therapeutic approach $[3,4]$. No prospective, randomized studies have yet been performed to evaluate its effectiveness and efficacy compared to the conventional therapy.

The CRPS outpatient department of the Radboud University Nijmegen Medical Centre has a long history regarding treatment and research on CRPS-1 [2-7].

\section{Magnitude of the problem}

The incidence of CRPS-1 in the Netherlands is estimated at around 26.2:100,000 person years and females are 3.4 times more effected than males [8]. About $22 \%$ of patients show long-lasting symptoms and signs [2]. About 30\% of CRPS-1 patients completely omit their work during more than one year. [9].

A vast proportion of CRPS-1 patients needs major adaptations of their homes to be able to function with their activity limitations and participation restrictions.

\section{Treatment of CRPS-1}

\section{Conventional therapy}

Current conventional therapy for CRPS-1 has been consolidated in the Dutch national clinical guideline of 2006 [10]. It is focused on the relief of symptoms and pain with pharmacological treatment and immobilization of the affected limb, supported by physical therapy (e.g. by increasing control of pain, improving skills and optimizing coping with CRPS-1) and occupational therapy (e.g. by improving functional abilities and independence in activities of daily living) [6]. It results in approximately 50\% reduction of disability and pain in patients with CRPS-1.

Pharmacological treatment consists of analgesics in a step-up procedure in accordance with the WHO's pain ladder. Dimethylsulphoxide 50\% ointment (DMSO) is prescribed as radical scavenger for initial treatment. In cases presenting with allodynia or hyperalgesia, gabapentine, amitryptilin or carbamazepine may be indicated. Dystonia, myoclonia and muscle spasms may be treated with baclofen, diazepam or clonazepam. Vasodilating drugs like verapamil, ketensin and pentoxiphyllin can be prescribed for patients presenting with cold skin. In persistent cold CRPS-1, thoracic sympathectomy can be considered as a treatment option [10].

\section{Pain exposure physical therapy}

PEPT is a functional form of physical therapy and consists of a progressive-loading exercise program and management of pain-avoidance behaviour without the use of specific CRPS-1 medication or analgesics. It is based on the assumption that behavioural and psychological factors can exacerbate pain and dysfunction and might help maintain the condition. Patients denominate clear treatment goals in the domains of activities and participation.

PEPT aims to decrease kinesiophobia, pain behaviour and learned non-use [11], and increase self-confidence in the patients' own physical possibilities. Living without adaptations or living independently from caregivers, returning to work and employment, and restarting domestic life, self-care, mobility, hobbies and sports in a short time are the main treatment goals. Pain relief itself is not a primary treatment goal, and patients are informed that an increase in pain during or after the exercises and activities might occur. Patients are reassured that an increase in pain is not a sign of injury or tissue damage. In this respect, all conscious and unconscious signs of catastrophizing and kinesiophobic behaviour are specified and talked through with the patient and partner. If, despite explanation, doubt remains about the treatment content or when patients are not motivated to act upon instructions of the therapists, the treatment will be ceased.

The treatment consists of progressive-loading exercises and desensitization beyond the patients' pain limits. To decrease the enhanced skin sensitivity for touch and pressure, desensitization is carried out using self-massage and forced use of the affected arm or leg in daily activities. The progressive-loading exercises are tailored and focused on specific body functions using standard techniques in regular physical therapy, including passive and active exercises to mobilize joints and muscle stretching. During progressive loading, the physical therapists act mainly as instructors, rewarding functional progression and providing schedules for exercises and activities at home. Complaining about pain is discouraged and it is no longer a subject of debate or a reason to reduce the treatment intensity. Partly due to a limited number of five sessions, PEPT is a very low-cost approach from micro- as well as macro-economic point of view.

\section{Rationales}

The pathophysiology of CRPS-1 is not well understood. Most of the studies that have been carried out are observational in nature, with small numbers of patients taking part and, in most cases, without a control group. The conclusions of the studies are not always direct observations, but tend to be interpretations of the observations made by the investigators. A distinction can be drawn between peripheral afferent, efferent and central mechanisms involved in the onset and continuance of CRPS-1. So far, no clear 
explanation has been found of how these various mechanisms are related to one another. Since no randomized controlled trial have been published yet to validate the different treatment strategies in patients with CRPS-1, the rationale for a specific treatment is considered to be sufficiently convincing to perform individual treatments in patients.

\section{Conventional therapy}

It seems that a number of peripheral afferent mechanisms may be involved in the pathophysiology of CRPS-1. Indications point to an inflammatory process, a neuroinflammatory process and tissue hypoxia. Anatomical changes have also been seen in CRPS-1 patients. If a (neuro)inflammatory process is involved, then immunological predisposition may be relevant. There are indications that immunological acquired and/or genetic susceptibility may be involved in the onset of CRPS-1 [12].

(Neuro) inflammation: Disorders in high energy phosphate metabolism in CRPS-1 patients [13], vascular leakage from macromolecules [14] and significantly higher concentrations of IL-6 and TNF alpha [15] and tryptase in fluid from artificially produced blisters in CRPS-1 patients, indicating mast cell activity [16], point to involvement of an inflammatory process in CRPS-1.

Arguments supporting the theory that neurogenic inflammation may play a role in triggering CRPS-1 are higher concentrations of bradykinin, neuropeptide Y, Calcitonin Gene-Related Peptide (CGRP) and vasoactive intestinal peptide in patients with CRPS-1 [17] and protein extravasation induced by substance P [18].

Tissue hypoxia: Some research findings point to tissue hypoxia in CRPS-1. Patients with the condition are more hyperalgesic to fluids of low $\mathrm{pH}$ than control subjects. This applies not only to the skin but also to the deeper somatic structures [19]. Patients with CRPS-1 have higher skin lactate levels than controls, suggesting a rise in anaerobic glycolysis as a consequence of chronic tissue hypoxia [20]. Capillary haemoglobin oxygenation of the skin is normal in control individuals, lower than normal in the affected limb following surgery, and lower than normal in both affected and control limbs of people with CRPS-1. This suggests skin hypoxia. Reduced blood circulation leading to under-nutrition of an affected limb may be a factor contributing to atrophy and ulceration [21].

Autonomic and motor dysfunctions: Autonomous symptoms are seen in 98\% of CRPS-1 patients, but often change as the condition progresses [22]. Autonomic functions described in connection with CRPS-1 are vasomotor and sudomotor changes, changes at transmitter level and changes at receptor level. The vasoconstrictive response of thermoregulating skin circulation is slower in all phases of CRPS-1, suggesting sympathetic denervation, which can lead to hypersensitivity to catecholamines in vascular structures [23]. Motor dysfunction is common in patients with CRPS-1. Besides muscle weakness, patients can develop 'neglect' syndrome. The limb feels strange (cognitive neglect), and mental and visual attention is needed to move a limb (motor neglect) [24].

\section{Pain exposure physical therapy}

Peripheral and central sensitization is a common feature in CRPS-1 as in other neuropathic pain syndromes [25]. Various studies have shown functional changes in patients with CRPS-1. Patients have altered central sensomotor response to tactile stimulation of the fingertip [26]. Referred sensations are a feature of CRPS-1, and this is evidence of central reorganization. Recent studies point to a crucial roleof the central nervous system (CNS) in the pathophysiology of CRPS-1 [27].

Not only the complex patterns of autonomic dysfunction, but also motor and sensory symptoms imply CNS alterations. Typically, active range of movement is restricted, whereas passive movement is often possible. Plastic CNS alterations might explain the complex sensory symptoms (e.g. glove-stocking sensory loss, 'foreign-hand' sensation, mislocalization after tactile stimulation, impaired perceptual learning ability). Central changes show both shifts in sensory and motor cortex representation of the limbs in CRPS-1. This altered reorganisation seems to be associated with a pain perception as in phantom limb pain. Therefore, a lack of cortical re-reorganization could be an important factor for long term pain $[28,29]$. Patients with CRPS- 1 show a significant reorganization of central motor circuits, with an increased activation of primary motor, parietal and supplementary motor cortices. In addition to fMRI studies, there are psychophysical studies showing that many patients with CRPS-1 suffer from cognitive and motor neglect-like symptoms. Summarizing these results, there is growing evidence that CNS alterations play an important role in the development and persistence of CRPS-1 [27].

Behavioural and psychological factors can exacerbate the pain and dysfunction in CRPS-1 and could help maintain the condition in some patients. Effective management of CRPS- 1 requires that these aspects be addressed as part of an integrated treatment approach [30].

\section{Current evidence}

\section{Conventional therapy}

Scientific argumentation for the physical therapy approach of CRPS-1 reveals a RCT with high level quality [6] indicating that physical therapy (in addition to medical treatment) has a clinically relevant positive effect on impairments of functions and pain. A systematic literature review [31] shows good to very good quality level II 
evidence that graded motor imagery is effective in reducing pain in adults with CRPS-1.

Analgesics are often used in clinical practice when treating patients with CRPS-1, and their use is described in various treatment protocols and guidelines [32-34]. The scientific support for their administration to patients with CRPS-1 is, however, very limited. Administration of standard analgesics appears to be based on experience in other fields. The administration sequence in Dutch practices is based on the Dutch national clinical guideline 2006, in accordance with the WHO's pain ladder [10]. Oral administration of analgesics is followed by intravenous administration or peripheral blockade techniques [35-39].

Dimethylsulphoxide $50 \%$ ointment (DMSO) has a much greater beneficial impact on CRPS-1 symptoms than placebo according to several studies $[40,41]$. Nacetylcysteine, at a dose of $600 \mathrm{mg}$ three times a day, was found to have a significantly better effect on primary cold CRPS-1 than DMSO ointment.

The anticonvulsant gabapentin causes a modest but significant reduction in neuropathic pain symptoms in CRPS-1 patients some weeks after the start of treatment $[42,43]$.

No randomized control studies have been carried out on orally administered muscle relaxants to treat the motor symptoms of CRPS-1 [44]. Benzodiazepines and high doses of baclofen could have a positive effect.

Intrathecal baclofen therapy (ITB) is an invasive therapy and should be limited to carefully selected patients and, because of a high complication rate, should be conducted only by physicians with considerable experience in the implantation and care of intrathecal devices $[45,46]$.

Muizelaar et al. investigated the effect of calciumchannel blockers in treating CRPS-1 [47]. They reported that they are most effective on CRPS-1 in the acute phase. However, the study is of moderate quality and size, and it is primarily descriptive. The outcomes were subjective, failing to describe the nature of the improvement in patients' conditions.

Sympathetic nerve blockade is an accepted treatment option for CRPS-1 patients; for the upper limb blockage of the stellate ganglion or the thoracic sympathetic nerves, for the lower limb the lumbar sympathetic nerves, conventionally carried out at L2 and L3. However, no randomized studies have been carried out on CRPS-1 patients comparing the effect of sympathetic blockades performed under local anaesthetics with valid controls.

\section{Pain exposure physical therapy}

Regarding intensive physical therapy, including more aggressive or painful exercises (desensitization, aerobics, sports and loading), we found three retrospective analyses in children with CRPS indicating that in up to $92 \%$ of the included children, a complete recovery of symptoms occurred after intensive physical therapy [48-50]. Watson et al. show the effectiveness and safety of an active "stress loading" program in 41 patients with reflex sympathetic dystrophy, with improvement in pain, trophic and vasomotor changes, range of motion and grip strength [51].

Pain Exposure Physical Therapy has been evaluated in a large case series of 106 patients with chronic CRPS-1 [4]. Ninety-four percent of the patients showed a significantly improved function of the affected extremity, of which $49 \%$ experienced full functional recovery. In addition, a significant reduction in the number of symptoms of CRPS-1, such as pain $(75 \%$ of patients improved), use of analgesics and aids, was registered. This case series suggests that PEPT is an effective and safe treatment for this group of patients, who are unresponsive to standard therapies.

We conducted a $n=1$ study on 20 patients with acute CRPS-1 to evaluate the safety of this approach in acute CRPS-1 [3]. None of the patients in this case series experienced an exacerbation of CRPS-1 during the follow-up period of 3, 6 and 12 months. They showed a significant improvement in functions and pain.

\section{Aim}

The aim of this study is to determine whether Pain Exposure Physical Therapy (PEPT) can improve functional outcomes in patients with CRPS-1.

\section{Methods/design}

\section{Design of the study}

This study is designed in accordance with the principles of a single-blinded, randomized clinical trial. After randomization to one of the two study groups, according to a pre-fixed scheme, baseline measurements (T0) are performed. Further measurements are taken during the course of the treatment at three months (T1) and at the end of the treatment at six months (T2). Follow up is at nine months (T3) after inclusion. Patients will undergo complete assessments at all time points.

Investigators performing the measurements will be blinded for the treatment group. Patients will be carefully instructed not to violate this blinding protocol.

This study is approved by the state regional ethical committee and is registered at www.clinicaltrials.gov with number NCT00817128 and at www.trialregister.nl with number NTR 2090.

\section{Setting}

All patients will be screened, randomized and treated in our university hospital, a level 1 trauma centre in a rural 
area of The Netherlands. Approximately 150 patients with suspected CRPS-1 are referred to our multidisciplinary outpatient department annually.

\section{Study eligibility criteria}

Hospitals and general practitioners in the region of our hospital will participate in recruitment of eligible patients. The diagnosis CRPS- 1 has to be confirmed by Bruehl/IASP criteria (see below) and screening for eligibility is between three and twenty-four months after initial injury. This screening will take place at the outpatient department of the Radboud University Nijmegen Medical Centre. All patients, between 18 and 80 years old, with CRPS-1 of the upper or lower extremity will then undergo eligibility assessment.

All patients with possible underlying diagnoses which may cause the pain syndrome will be excluded. Impairments of the contra-lateral extremity, relapse of CRPS-1, pregnancy, lactation and prior sympathectomy of the affected extremity are criteria for exclusion.

All patients have to give written informed consent prior to participation in this study. An information letter will be provided, depending on the treatment group.

\section{Modified research diagnostic criteria for CRPS}

1. Continuing pain, disproportionate to any inciting event

2. At least one symptom in each of the four following categories

a.Sensory: reports of hyperesthesia

b.Vasomotor: reports of temperature asymmetry and/or skin colour changes and/or skin colour asymmetry

c.Sudomotor/oedema: reports of oedema and/or sweating changes and/or sweating asymmetry d.Motor/trophic: reports of decreased range of motion and/or motor dysfunction (weakness, tremor, dystonia) and/or trophic changes (hair, nail, skin)

3. At least one sign in two or more of the following categories

a.Sensory: evidence of hyperalgesia (to pinprick)

and/or allodynia (to light touch)

b.Vasomotor: evidence of temperature asymmetry and/or skin colour changes and/or skin colour asymmetry

c.Sudomotor/oedema: evidence of oedema and/or sweating changes and/or sweating asymmetry d.Motor/trophic: evidence of decreased range of motion and/or motor dysfunction (weakness, tremor, dystonia) and/or trophic changes (hair, nail, skin)

4. The presence of an initiating noxious event, or a cause of immobilization
5. Symptoms cannot be explained by other diagnoses

\section{Sample size calculation}

The sample size calculation is based on the following finding and expectation. The improvement in the Impairment level SumScore (ISS), restricted version $[7,52]$, which is the primary outcome measure, for the conventional therapy group is $55 \%$ over one year [2]. For the PEPT-group it is estimated to be around $80 \%$ [4]. Given an alpha of 0.05 and a power of $80 \%$ for a one-sided Chi-square test, 62 patients are needed when using a 1:1 randomization, 31 in the PEPT-group and 31 in the conventional therapy group.

\section{Trial interventions}

\section{Conventional group}

The conventional treatment of CRPS-1, based on the recent Dutch guideline [10], includes pharmacological interventions with analgesics, $\mathrm{N}$-acetylcysteine, calcium channel blocker, ketanserine and dimethylsulphoxide for local application on the skin. In case of insufficient effect, sympathetic blockade, spinal cord stimulation and amputation may be considered. Following the Dutch guideline, patients in the conventional care group will also be referred to physical therapy to exercise the extremity in a pain contingent manner.

\section{Experimental group}

The experimental treatment is PEPT, a functional form of physical therapy combined with a cognitivebehavioural form of treatment. It consists of a maximum of five sessions of each $40 \mathrm{~min}$. Analgesics are contraindicated, the experienced pain will not be treated.

\section{Patient recruitment}

Diagnosis and treatment of CRPS-1 is performed by the multidisciplinary CRPS outpatient team, including a surgeon, a rehabilitation physician, a physical therapist, an anaesthesiologist and a research nurse. Around 150 new adult patients are visiting this outpatient clinic each year of which $23 \%$ is diagnosed having CRPS-1 (confirmed by Bruehl/IASP criteria) between 3 and 24 months after initial injury and without underlying diagnoses which may be responsible for the pain syndrome [53]. This means that around 40 patients with CRPS- 1 are diagnosed each year, who can be randomized accordingly. To increase the amount of eligible patients, the county hospitals as well as general practitioners in Nijmegen and surroundings will be asked to pre-screen and send patients with possible CRPS-1 to our outpatient department for screening and probable enrolment into the study. It is expected that this strategy will increase the amount of eligible patients. All these eligible patients will be 
screened by the multidisciplinary CRPS outpatient team prior to inclusion. Both the conventional treatment and PEPT will be executed in the Radboud University Nijmegen Medical Centre, with blinding of the observer during all measurements.

\section{Time schedule}

Patients will be screened and randomized from January 2009 until June 2011. A mean of 4 patients are planned to enrol each month once the study reaches full adhesion. After complete follow-up, it will be finished in the spring of 2012. The final report after data analysis will be expected at the end of 2012.

\section{Randomization}

Patients will be pre-screened, based on the referral letter sent by the county hospital or general practitioner, during a multi-disciplinary session of our team. In cases of potentially positive CRPS-1 in accordance with Bruehl/IASP criteria, these patients are invited to undergo thorough screening and multidisciplinary assessment by the same team. Patients with manifest CRPS-1 in accordance with the research diagnostic criteria of Bruehl/IASP, judged unanimously by the team, are asked to participate in the study. They are informed about the study by the research nurse and if they are interested, one week of consideration is scheduled. During this week, an accelerometer (PAM, physical activity monitor) is mounted on the affected wrist (CRPS-1 in the upper extremity) or affected ankle (CRPS-1 in the lower extremity) to measure the intensity of activity in T0. After this one week of consideration, a custom made computed randomization program is used to blindly allocate patients to one of the two treatment groups. The randomization program is made by an investigator with no clinical involvement in the trial.

Every patient who will be screened will specifically be informed about the possibility to change treatment group at any time after randomization, but preferably after having finished the initial allocated treatment. Once the computer randomization has been completed, patients who are allocated to the conventional care group are scheduled for their first treatment visit at the outpatient department of anaesthesiology within two weeks. Patients who are allocated to the PEPT group are scheduled for their first treatment at the outpatient department of rehabilitation within two weeks.

Each randomization package contains the following forms:

- Patient identification tabs

- Checklist hospital contacts

- Outpatient chart

- All written correspondence
- Travel compensation forms

- Randomization form

- Informed consent

- Measurement sets (mentioned under "outcome parameters") for all measure moments

- Diaries (physical activity, pain score and medication during seven days) for all measure moments

- Cost lists (e.g. doctor's visits, medical costs) for all measure moments

- Questionnaires (mentioned under "outcome parameters") for all measure moments

- PAM scoring list

- PAM result list

\section{Blinding procedure}

All measurements taken in both treatment groups during the course of this study are carried out by an experienced research nurse who underwent extensive measurement instructions, training and supervision before she was considered qualified to perform all measurements. She is blinded for the treatment group in all patients. In order to maintain this blindness, we thoroughly instruct all patients not to violate her blindness. To further guarantee her blindness, we instruct her to put a small amount of menthol ointment on her upper lip during each measurement session. This prevents her from smelling the garlic odour of DMSO ointment, which is part of the conventional treatment.

\section{Outcome parameters}

\section{Primary outcome measures}

The primary outcome measure is the Impairment level SumScore (ISS), restricted version [7,52]. The ISS, restricted version, consists of three measurement parameters (pain, active range of motion and temperature) and four measurement instruments (Visual Analogue Scale for Pain (VAS-P) [54], McGill Pain Questionnaire Dutch Language Version (MPQ-DLV) [55], goniometry of mobility of joints [56], and skin temperature) [57] and has a range of 4 to 40 points. Beside the ISS, restricted version, its individual components will be evaluated [52].

\section{Secondary outcome measures}

1. The Disability of the Arm, Shoulder and Hand (DASH), a questionnaire which maps both functions and daily activities of the upper limb $[58,59]$. The Lower-Limb Tasks Questionnaire (LLTQ) of McNair [60], a questionnaire which focuses on physical tasks related to function of the lower limb.

2. The fear-avoidance beliefs questionnaire (FABQ) [61], a statement list regarding the perception on pain and physical activities 
3. The level of participation as determined by the questionnaire SF-36 [62], which measures the patient's point of view regarding health

4. At function level, the muscle force measurements, as derived from both hands and feet by a handheld dynamometer (MicroFET) [63,64].

5. At level of activity limitations, for the legs the $10 \mathrm{~m}$ walking test [65] (which measures the time walking a certain distance) and the Timed Up and Go test $[66,67]$ (which measures the time from rising from a chair, walking a restricted distance to sitting down again).

6. Compliance and adherence: adherence to longterm therapy is defined by the World Health Organization as the extent to which a person's behaviour corresponds with agreed recommendations from a healthcare provider [68]. Physical activities like walking and exercises, combined with an adequate use of medication, aids and appliances, are essential to guarantee success in the present study on patients with CRPS-1. However, little is known about the level of physical activity, the intensity of exercises and the adherence of these patients to recommendations from the physician and physical therapist. Therefore, an extensive assessment will be performed by interview (about activities such as changing and maintaining body positions, carrying, handling and moving objects, walking and moving, self-care and household tasks, and exercises), questionnaires (the Seven Days Physical Activity Recall questionnaire (PAR) $[69,70]$, the International Physical Activity Questionnaire (IPAQ) [71], the Pain Catastrophizing Scale (PCS; a questionnaire regarding the perception of pain) [72] and the Pain Disability Index (PDI; a list measuring the influence of pain complaints on daily life) [73]) and an accelerometer (PAM) [74] to monitor compliance and adherence. The PAM will be used as a control device for over- or underreporting of physical activity via questionnaires (PAR, IPAQ). Patients will be instructed to wear the PAM from the moment they go out of bed in the morning until the moment they go to bed at the end of the day. Scores for self-reported physical activity will be combined with PAMscores to validate self-reported physical activity. Self-reported adherence with PEPT will be combined with observations of physicians and physical therapists.

7. Quality of life will be measured with the EuroQol (EQ-5D) [75]; this is on behalf of the economic evaluation
8. Adverse reactions will be monitored throughout the trial using standardized Serious Adverse Event forms, specifically regarding exacerbations of CRPS-1 signs and symptoms leading to medical consultation.

\section{Economic analysis}

This study investigates the potential efficiency of PEPT versus conventional treatment in patients with CRPS-1 from a societal perspective. The economic evaluation is based on the general principles of a cost-effectiveness analysis. Primary outcome measures for the economic evaluation are costs and quality adjusted life years (QALYs). The ratio cost per QALY gained (ICER, incremental cost effectiveness ratio) will be computed and uncertainty will be determined using the bootstrap method or Fieller method. Finally, a cost-effectiveness acceptability curve will be derived that is able to evaluate efficiency by using different thresholds (WTP, willingness to pay) for a QALY. The impact of uncertainty surrounding deterministic parameters (for example cost-prices) on the ICER will be explored using one-way sensitivity analyses on the range of extremes. The economic evaluation is being done alongside the clinical trial and consequently adheres to the earlier presented design and measurement points.

\section{Patient outcome analysis}

The effect analysis adheres to the design of a randomized controlled trial, relevant for the economic evaluation quality of life. Quality adjusted life years (QALYs) will be computed (using the trapezium rule) in order to perform a cost-utility analysis for the two alternative strategies. For the overall quantification of health status as a single index (utilities) we use the standard EQ-5D classification system developed by the EuroQol Group. The EQ-5D is one of the three widely used multi-attribute systems available to determine health states preferences (utilities). We chose the EQ-5D because 1) the five domains of the EQ-5D reflect aspects that are thought to be important for the population under consideration, 2) the system is relatively simple to administer, 3) the sensitivity of the instrument proved to be satisfactory, and 4) a reasonable sound algorithm has been published to compute utilities.

\section{Cost analysis}

The cost analysis exists of two main parts. First, on patient level, volumes of care will be measured prospectively using standardized Case Report Forms (CRF) and patient-based diaries. Per treatment group, full costprices will be determined using activity based costing. Activities in both production processes are outpatient visits and physical therapy. Medication costs constitute of analgesics, DMSO 50\%, n-acetylcysteine, gabapentine, carbamazepine, amitriptylin, nortriptylin, baclofen, diazepam, 
clonazepam and sympathetic blocks. Productivity losses for patients will be estimated using an interview on a 2 months recall basis by the researcher. The frictioncost-method will be applied. Also travel time to therapy or outpatient clinic and related costs will be considered, on the basis of 2 months recall.

The second part of the cost analysis consists of determining the cost prices for each volume of consumption in order to use these for multiplying the volumes registered for each participating patient. The Dutch guidelines for cost analyses will be used [76]. For units of care/resources where no guideline or standard prices are available, real cost prices will be determined.

The potential effects on medical costs are expected to be approximately $€ 2802,75$ per treatment in conventional care and around $€ 1008,50$ per treatment in PEPT. Costs based on social participation and work are not included in these prices (see Table 1).

\section{Statistical analysis}

All measured data will be assembled in a computer database and analyzed using SPSS 17.0. For all outcome variables, "effects" will be calculated at all evaluation time points during as well as after treatment, by taking values at T0 as reference values. Outcomes for each group will be plotted graphically in time to study their patterns. Linear mixed models will be used to examine possible differences over the course of time and to find out if these differences between treatment groups can be considered as statistical significant. For the level of significance alpha $=0.05$ will be used. As the difference between groups in ISS, restricted version, is expected to be around $25 \%$, we take this as a minimal clinically important difference (MCID) in effect between groups between T0 (intake) and T3 (follow-up) for the primary outcomes.

For the secondary outcomes, even as for cost differences, explorative tests will be used (Wilcoxon). Two analyses will be performed: an intention-to-treat analysis (ITT) and a per-protocol analysis (PP). In the ITT analysis, outcomes of all the participants will be used for the group they are originally assigned to. In the PP analysis, outcomes of protocol violators will be ignored.

\section{Discussion}

Clinical trials on the treatment of patients with CRPS-1 are scarce due to deficiency and controversy in objective diagnostic criteria and underlying pathophysiology. Most clinicians support an approach of multiple interventions at different dimensions, customized to the individual patient. Therefore, only few patients can be identified with a homogenous clinical presentation for whom a therapeutic approach can be standardized. Intervention trials in patients with CRPS-1 have therefore typically been focused on a

Table 1 Medical costs

\begin{tabular}{|c|c|c|c|c|}
\hline & Conventional therapy (CBO 2006) & Euro & Experimental therapy (PEPT) & Euro \\
\hline \multicolumn{5}{|l|}{ Medical costs } \\
\hline $\begin{array}{l}\text { Outpatient department } \\
\text { (first visit) }\end{array}$ & 1 time & 45 & 1 time & 45 \\
\hline $\begin{array}{l}\text { Outpatient department } \\
\text { (succeeding visits) }\end{array}$ & 6 times & 192 & 1 time & 32 \\
\hline $\begin{array}{l}\text { Physical therapy } \\
\text { (first session) }\end{array}$ & 1 time & 65 & $2(1 \times 2$ therapists $)$ & 130 \\
\hline $\begin{array}{l}\text { Physical therapy } \\
\text { (succeeding sessions) }\end{array}$ & 25 times & 1218.75 & $10(5 \times 2$ therapists $)$ & 487.50 \\
\hline Travel expenses & 33 times & 740 & 14 times & 314 \\
\hline \multicolumn{5}{|l|}{ Other medical costs } \\
\hline $\begin{array}{l}\text { Analgesics in accordance with } \\
\text { WHO-standard } \\
\text { (until step 2) }\end{array}$ & $\begin{array}{l}6 \text { months NSAID } \\
3 \text { months tramadol } \\
\text { Paracetamol }\end{array}$ & $\begin{array}{l}150 \\
90\end{array}$ & n.a. & n.a. \\
\hline DMSO 50\% & 3 months & 60 & n.a. & n.a. \\
\hline $\begin{array}{l}\text { n-acetylcysteïne } \\
\text { Gabapentine } \\
\text { Carbamazepine } \\
\text { Amitriptyline/Nortriptyline }\end{array}$ & $\begin{array}{l}6 \text { months á } € 80 \\
6 \text { months à } € 15 \\
6 \text { months à } € 5\end{array}$ & 200 & n.a. & n.a. \\
\hline \multicolumn{5}{|l|}{ Miscellaneous } \\
\hline $\begin{array}{l}\text { Baclofen; Diazepam; Clonazepam } \\
\text { Vasoactive agents }\end{array}$ & $\begin{array}{l}6 \text { months à } € 13 \times 0.1 \\
6 \text { months à } € 9 \times 0.4\end{array}$ & $\begin{array}{l}7 \\
10\end{array}$ & n.a. & n.a. \\
\hline $\begin{array}{l}\text { Sympathetic block } \\
\text { (per treatment) }\end{array}$ & $€ 125 \times 0.2$ & 25 & n.a. & n.a. \\
\hline Total costs per patient per year & & 2802.75 & & 1008.50 \\
\hline
\end{tabular}


single item of this approach. This makes the results of individual trials difficult to interpret and the comparison of multiple trials impossible. One of the most important efforts to improve the level of evidence for treatments in this field is to equalize diagnostic criteria. At present, the Bruehl/IASP criteria are the international standard to diagnose a patient with suspected CRPS-1, not only for research applications but also in clinical practice. In our outpatient department for CRPS-1 patients, we use these criteria without restraints ever since 2004. Clinical studies from The Netherlands have been highly respected internationally due to the large amounts of patients that are treated in our country. It has to be confirmed that the diagnostic criteria according to Veldman, which have been used nationwide, are no longer valid. More than 75\% of patients who are referred with positive Veldman criteria do not have CRPS-1 according to the Bruehl/ IASP criteria [53].

Due to the evolvement in diagnosing CRPS-1, it is possible to recruit a homogenous patient group, which is eligible for solid prognostic studies such as the current one.

The new and promising approach in the treatment of CRPS-1, PEPT, has proven to be safe and possibly effective, and is now ready for clinical evaluation.

This is the first randomized controlled study with single blinding that has ever been planned in patients with CRPS-1 and does not focus on one single item but compares treatment strategies based on completely different pathophysiological and cognitive theories.

\begin{abstract}
Abbreviations
CRPS-1: Complex Regional Pain Syndrome type 1; PEPT: Pain Exposure Physical Therapy; IASP: International Association for the Study of Pain; WHO: World Health Organization; DMSO: Dimethylsulphoxide 50\% ointment; CNS: Central nervous system; fMRI: Functional Magnetic Resonance Imaging; ISS: Impairment level SumScore; PAM: Physical activity monitor; QALY: Quality adjusted life year,
\end{abstract}

\section{Competing interests}

Financial competing interests.

The authors declare that they have no financial competing interests. Non-financial competing interests.

The authors declare that they have no non-financial competing interests.

\section{Authors' contributions}

KB largely rewrote the manuscript after critically revising it, and finished the manuscript. RO contributed substantially to conception and design and by revising it critically for important intellectual content. RvD assisted in study design and drafted the parts on conventional therapy. FK assisted in study design and drafted the parts on the experimental treatments. HS assisted in study design and drafted the parts on secondary outcome parameters. GJvdW assisted in study design and drafted the parts on power calculation and randomization. EA assisted in study design and drafted the parts on economical and cost analysis. HG drafted the parts on statistical analysis. HvdM conceived of the study, and participated in its design and coordination and helped to draft the manuscript. JPF originally drafted the manuscript in accordance with the awarded research grant from the Dutch Government (ZonMw). All authors read and approved the final manuscript.

\section{Authors' information}

KB is currently a medical student at the Radboud University Nijmegen. $\mathrm{RO}$ started his education in physical therapy (PT) at the Sint Maartenskliniek, Nijmegen, The Netherlands in 1963. He received his bachelor degree PT in 1966 and his degree in Manual Physical therapy (MPT) in 1972. He studied rehabilitation sciences (MSc) at the Free University of Brussels, Belgium which he finished in 1984 cum laude. Between 1984-1988 he did his PhD at the Radboud University Nijmegen, The Netherlands. Between 1968-1982 he was head of the department of physical therapy in general hospitals and teacher at the School of Physical Therapy 'West Brabant', Breda, The Netherlands. Between 1988-2007, he became research director of the Dutch Institute of Allied health Care, Amersfoort, The Netherlands. Between 1989-2000 he was full professor Manual Therapy at the Free university of Brussels, Belgium. From 2000 until his retirement in 2008, he was full professor in Allied Health Sciences at the Radboud University Nijmegen, The Netherlands, with a focus on the quality of allied health care and development, implementation and evaluation of clinical guidelines. He has a long-standing practical experience with regards to treatment of patients with Complex Regional Pain Syndrome (CRPS). He has published about 250 articles in national and international journals. He is member of various national and international physical therapy organizations and reviewer of national and international journals.

RvD is anaesthesiologist at the Pain Centre of the Radboud University Nijmegen Medical Centre, The Netherlands since 1988. He participates in all activities in an Academic Multidisciplinary Pain Centre for acute pain, chronic pain, pain in cancer and palliative care. Specific expertise in invasive pain treatment, neuromodulation for pain relief. He is Chairman of the Dutch Society for Neuromodulation and former Chairman of Pain Section of the Dutch Society for Anaesthesiology.

FK is working as a physical therapist since 33 years in his own practice and at the Department of Physical Therapy of the Radboud University Nijmegen Medical Centre. Specific expertise in treatment of patients with chronic pain and Complex Regional Pain Syndrome (CRPS). He is also working as a Quality of Care employee at the Department of Physical Therapy.

HS has been working as a clinical psychologist in pain medicine since 1990 and is working at the Academic Centre of Pain and Palliative Care of the Radboud University Nijmegen Medical Centre. He successfully finished his PhD thesis in 2008, titled Psychological factors in chronic pain: from predictors to implementation. In 2002 he wrote a book titled The psychologist as pain specialist. He is specialized in diagnosing and treating chronic pain within tailored treatment designs and in translating scientific knowledge into clinical practice for doctors, psychologists, physical therapists and nurses.

GJvdW is professor of Health Technology Assessment at the Radboud University Nijmegen Medical Centre and professor of Interactive Health Technology Assessment at the Athena Institute, Free University Medical Centre, Amsterdam. He is a member of the Standing Committee on Medical Technology Assessment of the Health Council of The Netherlands and member of the National Appraisal Committee at the National Health Insurance Board. He serves on the Editorial Board of the International Journal of Technology Assessment in Health Care and is a member of the International Network of Agencies for Health Technology Assessment (INAHTA).

EA studied graduated as an economist in 1990. Then a professional career was started as a university lecturer at Maastricht University, faculty of Economics. In 1997, he successfully finished his PhD thesis (promoter, prof. dr. G. Kootstra, surgeon). Earlier, in 1996, he started as an assistant professor at the department of Health, Organization, Policy, and Economics (BEOZ) of the faculty of Health Sciences, Maastricht University. In the beginning of 2001 he started as a senior consultant at the Ministry of Economic Affairs in The Hague, which he left late 2001 for an assistant professorship at the Radboud University Nijmegen Medical Centre. Here, he joined the department of Medical Technology Assessment of which he became deputy head in 2004. Presently the department is merged into the department of Epidemiology, Biostatistics and HTA. Several publications about economical aspects of health care are from his hand.

HG studied Biology and Biophysics at the Radboud University Nijmegen (Msc; 1985). In 1986, after additional training in information technology, he joined the department of Epidemiology as a scientific programmer. He specialized in methodological and statistical support of several PhD projects. He also lectured in statistical methods for Biomedical students. From 2005 on, he specialized in Health Technology Assessment. Nowadays, he 
participates in several research projects of major departments at the Radboud University Nijmegen Medical Centre as a methodological and statistical consultant

HvdM is consultant at the Department of Rehabilitation Medicine at the Radboud University Nijmegen Medical Centre, The Netherlands. He worked at the department of neurosurgery Erasmus UMC Rotterdam and AMC Amsterdam, both in The Netherlands. He worked as post-doc at the Institute for Brainresearch Zurich, Switzerland and as house officer neurology both at the University Clinic Balgrist Zurich, Switzerland and at the NRZ Greifswald in Germany. He was trained as rehabilitation physician at the Sint Maartenskliniek in Nijmegen, The Netherlands. Since 2003, he is associate professor at the Department of Rehabilitation Medicine at the Radboud University Nijmegen Medical Centre, The Netherlands with specialization in care, research and education in management of spasticity in children with cerebral palsy, acute management and rehabilitation of spinal cord injury, development of innovative orthotics and prosthesis for trauma and orthopaedic rehabilitation, early intensive physical and cognitive rehabilitation of patients with critical illness with application of virtual reality devices (X-VR-D) and experimental management and rehabilitation of patients with CRPS-1. He cooperates in the multidisciplinary outpatient department for patients with CRPS since 2004.

JPF is consultant (general and orthopaedic) trauma surgeon at the Department of Surgery from the Radboud University Nijmegen Medical Centre in The Netherlands. He graduated from The University of Utrecht Medical School, The Netherlands in 1990 with a specialization in General Surgery in 1996 and a subspecialization in Trauma Surgery in 2001 at the Free University Medical Centre in Amsterdam, The Netherlands. In the same year he defended his thesis successfully. In 2009, he graduated in clinical epidemiology at the Radboud University Nijmegen, The Netherlands. In 2004, he adopted the outpatient department for CRPS patients from his famous predecessor professor dr. RJA Goris, who retired at that time.

Received: 11 January 2012 Accepted: 19 April 2012

Published: 19 April 2012

\section{References}

1. Bruehl S, Harden RN, Galer BS, Saltz S, Bertram M, Backonja M, Gayles R, Rudin N, Bhugra MK, Stanton-Hicks M: External validation of IASP diagnostic criteria for Complex Regional Pain Syndrome and proposed research diagnostic criteria. International Association for the Study of Pain. Pain 1999, 81:147-154.

2. Vaneker M, Wilder-Smith $\mathrm{OH}$, Schrombges $P$, Oerlemans HM: Impairments as measured by ISS do not greatly change between one and eight years after CRPS 1 diagnosis. Eur J Pain 2006, 10:639-644.

3. Van de Meent H, Oerlemans M, Bruggeman A, Klomp F, van Dongen R, Oostendorp R, Frölke JP: Safety of "pain exposure" physical therapy in patients with complex regional pain syndrome type 1. Pain 2011, 152(6):1431-1438.

4. Ek JW, van Gijn JC, Samwel H, van Egmond J, Klomp FP, van Dongen RT: Pain exposure physical therapy may be a safe and effective treatment for longstanding complex regional pain syndrome type 1: a case series. Clin Rehabil 2009, 23(12):1059-1066.

5. Veldman PH, Reynen HM, Arntz IE, Goris RJ: Signs and symptoms of reflex sympathetic dystrophy: prospective study of 829 patients. Lancet 1993, 342:1012-1016.

6. Oerlemans HM, Oostendorp RA, de Boo T, Goris RJ: Pain and reduced mobility in complex regional pain syndrome I: outcome of a prospective randomised controlled clinical trial of adjuvant physical therapy versus occupational therapy. Pain 1999, 83:77-83.

7. Oerlemans HM, Goris RJ, Oostendorp RA: Impairment level sumscore in reflex sympathetic dystrophy of one upper extremity. Arch Phys Med Rehabil 1998, 79(8):979-990.

8. De Mos M, de Bruijn AG, Huygen FJ, Dieleman JP, Stricker BH, Sturkenboom MC: The incidence of complex regional pain syndrome: a population-based study. Pain 2007, 129(1-2):12-20.

9. Geertzen JH, Dijkstra PU, Groothoff JW, ten Duis HJ, Eisma WH: Reflex sympathetic dystrophy of the upper extremity- a 5.5-year follow up. Part II. Social life events, general health and changes in occupation. Acta Orthop Scand Supp/ 1998, 279:19-23.
10. Perez RS, Zollinger PE, Dijkstra PU, Thomassen-Hilgersom IL, Zuurmond WW, Rosenbrand KC, Geertzen JH; CRPS I task force: Evidence based guidelines for complex regional pain syndrome type 1. BMC Neurol 2010, 10:20.

11. Taub E, Uswatte G, Mark W, Morris DM: The learned nonuse phenomenon: implications for rehabilitation. Eura Medicophys 2006, 42(3):241-256.

12. De Rooij AM, Florencia Gosso M, Haasnoot GW, Marinus J, Verduijn W, Claas $\mathrm{FH}$, van den Maagdenberg AM, van Hilten JJ: HLA-B62 and HLA-DQ8 are associated with Complex Regional Pain Syndrome with fixed dystonia. Pain 2009, 145:82-85.

13. Heerschap A, den Hollander JA, Reynen H, Goris RJ: Metabolic changes in reflex sympathetic dystrophy: a 31P NMR spectroscopy study. Muscle Nerve 1993, 16(4):367-373.

14. Oyen WJ, Arntz IE, Claessens RM, van der Meer JW, Corstens FH, Goris RJ: Reflex sympathetic dystrophy of the hand: an excessive inflammatory response? Pain 1993, 55(2):151-157.

15. Huygen FJ, de Bruijn AG, de Bruin MT, Groeneweg JG, Klein J, Zijlstra FJ: Evidence for local inflammation in complex regional pain syndrome type 1. Mediators Inflamm 2002, 11(1):47-51.

16. Huygen FJ, Ramdhani N, van Toorenenbergen A, Klein J, Zijlstra FJ: Mast cells are involved in inflammatory reactions during Complex Regional Pain Syndrome type 1. Immunol Lett 2004, 91(2-3):147-154.

17. Calder JS, Holten I, McAllister RM: Evidence for immune system involvement in reflex sympathetic dystrophy. J Hand Surg Br 1998, 23(2):147-150

18. Schinkel C, Gaertner A, Zaspel J, Zedler S, Faist E, Schuermann M: Inflammatory mediators are altered in the acute phase of posttraumatic complex regional pain syndrome. Clin J Pain 2006, 22:235-239.

19. Birklein F, Weber M, Ernst M, Riedl B, Neundörfer B, Handwerker HO: Experimental tissue acidosis leads to increased pain in complex regional pain syndrome (CRPS). Pain 2000, 87(2):227-234.

20. Birklein F, Weber M, Neundörfer B: Increased skin lactate in complex regional pain syndrome: evidence for tissue hypoxia? Neurology 2000, 55(8):1213-1215.

21. Koban M, Leis S, Schultze-Mosgau S, Birklein F: Tissue hypoxia in complex regional pain syndrome. Pain 2003, 104(1-2):149-157.

22. Birklein F, Riedl B, Sieweke N, Weber M, Neundörfer B: Neurological findings in complex regional pain syndromes-analysis of 145 cases. Acta Neurol Scand 2000, 101(4):262-269.

23. Kurvers HA, Jacobs MJ, Beuk RJ, van den Wildenberg FA, Kitslaar PJ, Slaaf DW, Reneman RS: Reflex sympathetic dystrophy: result of autonomic denervation? Clin Sci 1994, 87(6):663-669.

24. Galer BS, Jensen M: Neglect-like symptoms in complex regional pain syndrome: results of a self-administered survey. J Pain Symptom Manage 1999, 18(3):213-217.

25. Nickel FT, Maihöfner C: Current concepts in pathophysiology of CRPS I. Handchir Mikrochir Plast Chir 2010, 42:8-14.

26. Juottonen $\mathrm{K}$, Gockel M, Silén T, Hurri H, Hari R, Forss N: Altered central sensorimotor processing in patients with complex regional pain syndrome. Pain 2002, 98(3):315-323.

27. Maihöfner C, Nickel FT, Seifert F: Neuropathic pain and neuroplasticity in functional imaging studies. Schmerz 2010, 24(2):137-145.

28. Moseley $\mathrm{GL}$ : Why do people with complex regional pain syndrome take longer to recognize their affected hand? Neurology 2004, 62(12):2182-2186.

29. Flor H: [Visualisation of phantom- and backpain using imaging techniques. Implication for treatment]. Orthopade 2004, 33(5):553-557.

30. Bruehl S, Chung OY: Psychological and behavioral aspects of complex regional pain syndrome management. Clin J Pain 2006, 22:430-437.

31. Daly AE, Bialocerkowski AE: Does evidence support physiotherapy management of adult Complex Regional Pain Syndrome Type One? A systematic review. Eur J Pain 2009, 13(4):339-353.

32. Stanton-Hicks M, Baron R, Boas R, Gordh T, Harden N, Hendler N, Koltzenburg M, Raj P, Wilder R: Complex Regional Pain Syndromes: guidelines for therapy. Clin J Pain 1998, 14(2):155-166.

33. Stanton-Hicks MD, Burton AW, Bruehl SP, Carr DB, Harden RN, Hassenbusch SJ, Lubenow TR, Oakley JC, Racz GB, Raj PP, Rauck RL, Rezai AR: An updated interdisciplinary clinical pathway for CRPS: report of an expert panel. Pain Pract 2002, 2(1):1-16.

34. Raja SN, Grabow TS: Complex regional pain syndrome I (reflex sympathetic dystrophy). Anesthesiology 2002, 96(5):1254-1260. 
35. Rico H, Merono E, Gomez-Castresana F, Torrubiano J, Espinos D, Diaz P. Scintigraphic evaluation of reflex sympathetic dystrophy: comparative study of the course of the disease under two therapeutic regimens. Clin Rheumatol 1987, 6(2):233-237.

36. Harke H, Gretenkort P, Ladleif HU, Rahman S, Harke O: The response of neuropathic pain and pain in complex regional pain syndrome I to carbamazepine and sustained-release morphine in patients pretreated with spinal cord stimulation: a double-blinded randomized study. Anesth Analg 2001, 92(2):488-495.

37. Connelly NR, Reuben S, Brull SJ: Intravenous regional anesthesia with ketorolac-lidocaine for the management of sympathetically-mediated pain. Yale J Biol Med 1995, 68(3-4):95-99.

38. Azad SC, Beyer A, Römer AW, Galle-Röd A, Peter K, Schöps P: Continuous axillary brachial plexus analgesia with low dose morphine in patients with complex regional pain syndromes. Eur J Anaesthesiol 2000, 17(3):185-188

39. Price DD, Long S, Wilsey B, Rafii A: Analysis of peak magnitude and duration of analgesia produced by local anesthetics injected into sympathetic ganglia of complex regional pain syndrome patients. Clin J Pain 1998, 14(3):216-226

40. Zuurmond WW, Langendijk PN, Bezemer PD, Brink HE, de Lange JJ, van Loenen AC: Treatment of acute reflex sympathetic dystrophy with DMSO 50\% in a fatty cream. Acta Anaesthesiol Scand 1996, 40(3):364-367.

41. Perez RS, Zuurmond WW, Bezemer PD, Kuik DJ, van Loenen AC, de Lange JJ, Zuidhof AJ: The treatment of complex regional pain syndrome type I with free radical scavengers: a randomized controlled study. Pain 2003, 102(3):297-307.

42. Serpell MG: Gabapentin in neuropathic pain syndromes: a randomised, double-blind, placebocontrolled trial. Pain 2002, 99:557-566.

43. Van de Vusse AC, Stomp-van den Berg SG, Kessels AH, Weber WE: Randomised controlled trial of gabapentin in Complex Regional Pain Syndrome type I [ISRCTN84121379]. BMC Neurol 2004, 4:13.

44. Van Hilten JJ: Movement disorders in complex regional pain syndrome. Pain Med 2010, 11(8):1274-1277.

45. Van Rijn MA, Munts AG, Marinus J, Voormolen JH, de Boer KS, Teepe-Twiss IM, van Dasselaar NT, Delhaas EM, van Hilten JJ: Intrathecal baclofen for dystonia of complex regional pain syndrome. Pain 2009, 143:41-47.

46. Raja SN: Motor dysfunction in CRPS and its treatment. Pain 2009, 143:3-4.

47. Muizelaar JP, Kleyer M, Hertogs IA, DeLange DC: Complex regional pain syndrome (reflex sympathetic dystrophy and causalgia): management with the calcium channel blocker nifedipine and/or the alpha-sympathetic blocker phenoxybenzamine in 59 patients. Clin Neurol Neurosurg 1997, 99(1):26-30.

48. Murray CS, Cohen A, Perkins T, Davidson JE, Sills JA: Morbidity in reflex sympathetic dystrophy. Arch Dis Child 2000, 82(3):231-233.

49. Sherry DD, Wallace CA, Kelley C, Kidder M, Sapp L: Short- and long-term outcomes of children with complex regional pain syndrome type I treated with exercise therapy. Clin J Pain 1999, 15:218-223.

50. Stanton RP, Malcolm JR, Wesdock KA, Singsen BH: Reflex sympathetic dystrophy in children: an orthopedic perspective. Orthopedics 1993, 16(7):773-779

51. Watson HK, Carlson L: Treatment of reflex sympathetic dystrophy of the hand with an active "stress loading" program. J Hand Surg Am 1987, 12(5 Pt 1):779-785.

52. Perez RS, Keijzer C, Bezemer PD, Zuurmond WW, de Lange JJ: Predictive value of symptom level measurements for complex regional pain syndrome type I. Eur J Pain 2005, 9:49-56.

53. Frölke JP, van Rumund A, de Waardt D, van Dongen RT, Klomp FP, Verbeek $A L$, van de Meent $H$ : Complex regional pain syndrome type 1 ? In $77 \%$ of people had a different diagnosis. Ned Tijdschr Geneeskd 2009, 153(12):550-553.

54. Breivik EK, Björnsson GA, Skovlund E: A comparison of pain rating scales by sampling from clinical trial data. Clin J Pain 2000, 16(1):22-28.

55. Vanderiet $\mathrm{K}$, Adriaensen $\mathrm{H}$, Carton H, Vertommen H: The McGill Pain Questionnaire constructed for the Dutch language (MPQ-DV). Preliminary data concerning reliability and validity. Pain 1987, 30(3):395-408.

56. Norkin CC, White DJ: Measurement of joint motion. A guide to goniometry. Philadelphia: F.A. Davis Company; 2009.

57. Bruggeman AW, Oerlemans MH, Frölke JP: Warm and cold complex regional pain syndromes: differences beyond skin temperature? Neurology 2009, 73(20):1711.
58. Veehof MM, Sleegers EJ, van Veldhoven $\mathrm{NH}$, Schuurman AH, van Meeteren $\mathrm{NL}$ : Psychometric qualities of the Dutch language version of the Disabilities of the Arm, Shoulder, and Hand questionnaire (DASH-DLV). $J$ Hand Ther 2002, 15(4):347-354.

59. Beaton DE, Katz JN, Fossel AH, Wright JG, Tarasuk V, Bombardier C: Measuring the whole or the parts? Validity, reliability, and responsiveness of the Disabilities of the Arm, Shoulder and Hand outcome measure in different regions of the upper extremity. J Hand Ther 2011, 14(2):128-146.

60. McNair PJ, Prapavessis H, Collier J, Bassett S, Bryant A, Larmer P: The lower-limb tasks questionnaire: an assessment of validity, reliability, responsiveness, and minimal important differences. Arch Phys Med Rehabil 2007, 88(8):993-1001.

61. Inrig T, Amey B, Borthwick C, Beaton D: Validity and Reliability of the Fear-Avoidance Beliefs Questionnaire (FABQ) in Workers with Upper Extremity Injuries. J Occup Rehabil 2009, 19:142-154.

62. Ware JE Jr: Sherbourne CD: The MOS 36-iten Short-Form Health Survey (SF-36). I. Conceptual Framework and Item Selection. Med Care 1992, 30(6):473-483.

63. Bohannon RW: Test-retest reliability of hand-held dynamometry during a single session of strength assessment. Phys Ther 1986, 66(2):206-209.

64. Bohannon RW, Andrews AW: Interrater reliability of hand-held dynamometry. Phys Ther 1987, 67(6):931-933.

65. Rossier P, Wade DT: Validity and reliability comparison of 4 mobility measures in patients presenting with neurologic impairment. Arch Phys Med Rehabil 2001, 82(1):9-13.

66. Schoppen T, Boonstra A, Groothoff JW, de Vries J, Göeken LN, Eisma WH: The Timed "up and go" test: reliability and validity in persons with unilateral lower limb amputation. Arch Phys Med Rehabil 1999, 80(7):825-828

67. Steffen TM, Hacker TA, Mollinger L: Age- and gender-related test performance in community-dwelling elderly people: Six-Minute Walk Test, Berg Balance Scale, Timed Up \& Go Test, and gait speeds. Phys Ther 2002, 82(2):128-137.

68. Adherence to long-term therapies - evidence for action [http://www.who. int/chp/knowledge/publications/adherence_introduction.pdf]

69. Blair SN, Haskell WL, Ho P, Paffenbarger RS Jr, Vranizan KM, Farquhar JW, Wood PD: Assessment of habitual physical activity by a seven-day recall in a community survey and controlled experiments. Am J Epidemiol 1985 122:794-804.

70. Sallis JF, Buono MJ, Roby JJ, Micale FG, Nelson JA: Seven-day recall and other physical activity self-reports in children and adolescents. Med Sci Sports Exerc 1993, 25(1):99-108.

71. Sjostrom M, Bull F, Craig C: Towards standardized global assessment of health-related physical activity the International Physical Activity Questionnaire (IPAQ). Med Sci Sports Exerc 2002, 34(5):S202.

72. Sullivan MJ, Bishop SR, Pivik J: The Pain Catastrophizing Scale: development and validation. Psychol Assess 1995, 7:524-532.

73. Pollard CA: Preliminary validity study of the pain disability index. Percept Mot Skills 1984, 59(3):974

74. Slootmaker SM, Chin A, Paw MJ, Schuit AJ, van Mechelen W, Koppes LL: Concurrent validity of the PAM accelerometer relative to the MTI Actigraph using oxygen consumption as a reference. Scand J Med Sci Sports 2009, 19(1):36-43.

75. Lamers LM, Stalmeier PF, McDonnell J, Krabbe PF, van Busschbach J.: Measuring the quality of life in economic evaluations: the Dutch EQ-5D tariff. Ned Tijdschr Geneeskd 2005, 149(28):1574-1578.

76. Hakkaart-van Roijen L, Tan SS, Bouwmans CAM: Handleiding voor kostenonderzoek, methoden en standaard kostprijzen voor economische evaluaties in de gezondheidszorg. College voor zorgverzekeringen. Geactualiseerde versie 2010.

doi:10.1186/1471-2474-13-58

Cite this article as: Barnhoorn et al:: The effectiveness and cost evaluation of pain exposure physical therapy and conventional therapy in patients with complex regional pain syndrome type 1. Rationale and design of a randomized controlled trial. BMC Musculoskeletal Disorders 2012 13:58 